\title{
QGA based MC-CDMA Detector
}

\author{
Ahmed M. Sana \\ Electrical Engineering \\ Department, College of \\ Engineering, University of \\ Baghdad, Baghdad, Iraq
}

\author{
Mohammed Nadhim \\ Abbas \\ Electrical Engineering \\ Department, College of \\ Engineering, University of \\ Baghdad, Baghdad, Iraq
}

\author{
Saleem M. R. Taha \\ Electrical Engineering \\ Department, College of \\ Engineering, University of \\ Baghdad, Baghdad, Iraq
}

\begin{abstract}
Multi-Carrier Code Division Multiple Access is regarded as one of the main candidates for $4 \mathrm{G}$ broadband wireless services. It combines the benefits of robustness to the multipath effects in OFDM systems and high privacy and security in CDMA systems. However, a problem arises in the detection of transmitted information due to the effect of noise, fading and other multipath effects. In this paper, a solution is proposed to this problem by introducing the quantum genetic algorithm based MC-CDMA detector. The performance of the proposed detector was investigated and compared with QGA based MIMO-OFDM detector proposed in previous work. The simulation results showed that the proposed QGA based MCCDMA detector was better in BER performance than QGA based MIMO-OFDM detector showing a performance closer to the optimum.
\end{abstract}

\section{Keywords}

MIMO-OFDM,MC-CDMA,QGA

\section{INTRODUCTION}

In the past decade, the world witnessed an increased demand on high speed, spectral efficient, secure, and low cost communication systems. For this purpose, many systems and techniques were proposed to perform this objective. One of the promising techniques that were proposed to perform this objective was the orthogonal frequency division multiplexing (OFDM) system. Use of OFDM technique makes the system more robust to the multipath effects and more spectral efficient since the bandwidth of the communication system is divided into small overlapping sub-carriers, which saves much bandwidth and reduces the effects of the multipath propagation.

Code division multiple access (CDMA) technique was also proposed. CDMA system has many advantages such as privacy of transmission, resistance to fading, and jamming and interference avoiding [1]. Recently, the MC-CDMA system was proposed to combine the benefits of the CDMA systems with the natural robustness to the frequency selective fading offered by OFDM [2]. However, a problem arises in the detection of the transmitted signals of such system due to the effect of the noise, fading, and multipath effects, which necessitates the use of an equalizing technique to equalize the effect of the wireless channel. A solution was proposed to this problem by introducing the quantum genetic algorithm based MC-CDMA detector, which uses the principles of quantum computing to find the solution.

In this paper, a theoretical analysis will be given for the MCCDMA systems and quantum genetic algorithms in section II and III, the structure of QGA based MC-CDMA detector and the procedure by which the QGA follows to retrieve the transmitted signal will be discussed in IV, the simulation results of the proposed MC-CDMA detector will be given in section $\mathrm{V}$, and finally, the most important conclusions about the proposed MC-CDMA detector proposed in this paper will be discussed in section VI.

\section{MC-CDMA SYSTEM}

Multi-Carrier Code Division Multiple Access (MC-CDMA) is a multiple access scheme used in OFDM-based telecommunication systems, allowing the system to support multiple users at the same time [3]. It is composed of two main sub-systems. The first system is the DS-CDMA system where the data streams from individual users are spread, summed, and fed to the second system represented by the OFDM system. In the OFDM stage, the data will modulate orthogonal sub-carriers to be broadcasted through the channel. The two stages of the MC-CDMA system will be explained in detail and discussed.

\section{DS-CDMA STAGE}

DS-CDMA is a multi access technique where multiple users access the shared medium using the same bandwidth at the same time but via different orthogonal codes [1]. These orthogonal codes are high rate streams, which are used to spread the users data over a wide spectrum. This is done by multiplying each user data stream by unique code in order to perform the spreading. Many orthogonal codes sets can be used for spreading in CDMA systems, such as Walsh codes set and Gold codes set [1]. However, the Walsh codes set is the most common set used in CDMA systems. Fig (1) shows the block diagram of CDMA transmission stage.

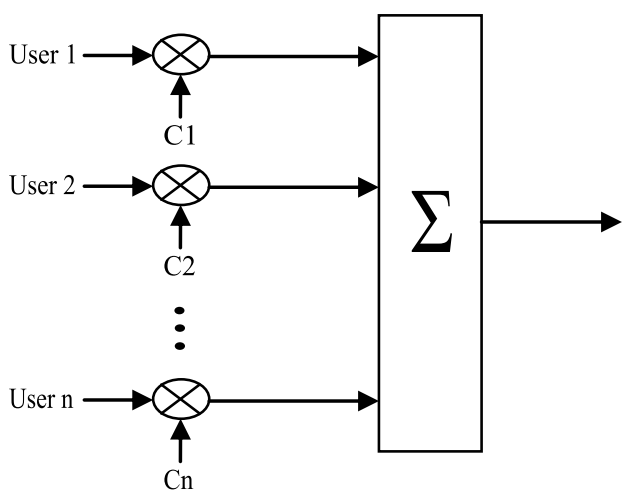

Figure 1:Block Diagram of CDMA Stage 
As shown in fig.(1), the data from multiple users are multiplied by high rate codes in order to spread them over wide spectrum. Spreading the data of users has the advantage of increased link security and decreased probability of jamming or detection since the data cannot be extracted or interfered without knowing the spreading code. After spreading process, the spread data from multiple users are summed and fed to the OFDM modulator stage for further process.

\section{OFDM STAGE}

After the CDMA stage is completed, the output data is encoded, mapped by a certain mapping scheme, and converted from serial form to parallel form via $\mathrm{S} / \mathrm{P}$ converter. After conversion to parallel streams, data sub-streams are fed to the IFFT block, so the parallel stream will modulate an overlapping orthogonal sub-carriers. This modulation has the advantage of decreasing the effect of multipath propagation since the bandwidth of the sub-carrier is narrow that it will approximately experience a flat fading, This will lead to a big simplification in the detection process at the receiver. In addition to the robustness to the multipath effects, the modulation of data on orthogonal sub-carriers saves much bandwidth since the sub-carriers are allowed to overlap, which increases the spectral efficiency of the system. After the IFFT process completes, the modulated streams are converted to serial form via $\mathrm{P} / \mathrm{S}$ converter. Cyclic prefix is added to the packet to eliminate any possible ISI or ICI, then the resulted signal is broadcasted through the channel. Fig (2) shows the structure of OFDM transmitter.

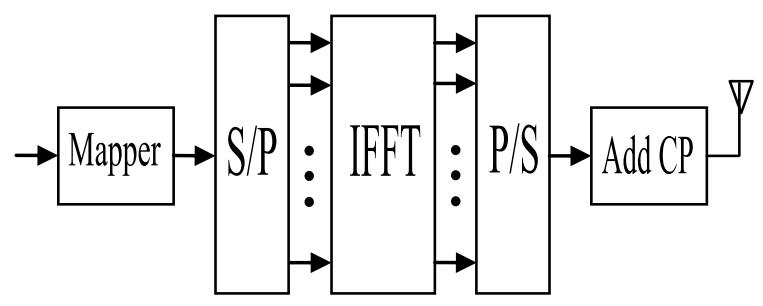

Figure 2: Structure of OFDM Stage

\section{QUANTUM GENETIC ALGORITHM}

Many representations can be used to encode the solutions onto individuals in the QGA. However, there are three types of representation which are : binary, numeric, and symbolic. The QGA uses another kind of representation, which is the Q-gene representation. It was called for probabilistic representation based on quantum bits [4].

The quantum bit is defined as the smallest unit of information stored as 0 or 1 or the superposition of the two [5]. The expression for the qubit is shown in eq.(1) .

$$
|\psi>=\alpha| 0>+\beta \mid 1>
$$

Where $(\alpha, \beta)$ or $[\alpha \beta]^{\mathrm{T}}$ are the operators of the quantum bits, so the value of $|\alpha|^{2}$ represents the probability that the qubit is found in 0 while $|\beta|^{2}$ represent the probability that the qubit will be found in 1 .

The quantum chromosome ore Q-chromosome is a set of Qgenes which represented as shown in eq.(2) .

$$
\left[\begin{array}{llll}
\alpha_{1} & \alpha_{2} & \cdots & \alpha_{k} \\
\beta_{1} & \beta_{2} & \ldots & \beta_{k}
\end{array}\right]
$$

Where $\alpha_{i}^{2}+\beta_{i}^{2}=1, \mathrm{i}=1,2,3, \ldots \mathrm{k}$

The Q-gene representation has the advantage that is able to represent a linear superposition of states. As example if Qchromosome with three pairs of amplitudes is assumed as shown in eq.(3).

$$
\left[\begin{array}{lll}
1 / \sqrt{2} & \sqrt{3} / 2 & 1 / 2 \\
1 / \sqrt{2} & 1 / 2 & \sqrt{3} / 2
\end{array}\right]
$$

From equations (2) and (3), it can be concluded that the probabilities for the states $|000>| 001>,,|010>| 011>$,, $|100>| 101>,, \mid 110>$, and $\mid 111>$ are $3 / 32,9 / 32,1 / 32$, $3 / 32,3 / 32,9 / 32,1 / 32$, and $3 / 32$ respectively which means that the 3 Q-genes chromosome was able to represent 8 states. While in conventional genetic algorithm, it will needed 8 strings to represent the eight states explained above.

The QGA uses quantum gates to make the chromosomes converge towards the optimum solution. The quantum gate is defined as the variation operators of QGA by which the updated gene should satisfy the normalization condition[4,5].

One of the important quantum gates that may be used in this work is the rotation gate which is expressed as shown in eq.(4).

$$
U(\emptyset)=\left[\begin{array}{cc}
\cos \emptyset & -\sin \emptyset \\
\sin \emptyset & \cos \emptyset
\end{array}\right]
$$

This gate is used to bias the gene towards 1 or 0 depending on the sign of the rotation angle $\phi[6]$. The rotation angle can be expressed as shown in eq.(5).

$$
\emptyset=k . f\left(\alpha_{i}, \beta_{i}\right)
$$

Where $\mathrm{k}$ is a factor whose value affects the speed of solution convergence. If the $\mathrm{k}$ was too small, the QGA may take much time to converge to the optimum solution which adversely affects on the performance of QGA. While choosing large values will cause the QGA converge or diverge to a prematurely local optimum, which may lead to errors. However, most designers take the rotation angle value in the range of $0.005 \pi-0.05 \pi[6,7]$.

$\mathrm{f}\left(\alpha_{\mathrm{i}}, \beta_{\mathrm{i}}\right)$ on the other hand represents the rotation angle sign. It can be obtained using the look up table shown in Table (1) :-

Table (1) :- Look up table for function $f(\alpha, \beta)$ [5]

\begin{tabular}{|c|c|c|c|}
\hline $\mathbf{d}_{\mathbf{1}}>\mathbf{0}$ & $\mathbf{d}_{\mathbf{2}}>\mathbf{0}$ & \multicolumn{2}{|c|}{$\mathbf{f}(\boldsymbol{\alpha}, \boldsymbol{\beta})$} \\
\cline { 3 - 4 } & & $\boldsymbol{\mu}_{\mathbf{1}}>\boldsymbol{\mu}_{\mathbf{2}}$ & $\boldsymbol{\mu}_{\mathbf{1}}<\boldsymbol{\mu}_{\mathbf{2}}$ \\
\hline True & True & +1 & -1 \\
\hline True & False & -1 & +1 \\
\hline False & True & -1 & +1 \\
\hline False & False & +1 & -1 \\
\hline
\end{tabular}

Where $\mathbf{d}_{\mathbf{1}}=\alpha_{1} \cdot \beta_{1}, \mathbf{d}_{\mathbf{2}}=\alpha_{2} \cdot \beta_{2}, \boldsymbol{\mu}_{\mathbf{1}}=\tan ^{-1}\left(\alpha_{1} / \beta_{1}\right), \boldsymbol{\mu}_{\mathbf{2}}=\tan ^{-1}\left(\alpha_{2}\right.$ $\left./ \beta_{2}\right), \boldsymbol{\alpha}_{1}, \boldsymbol{\beta}_{1}$ is the probability amplitude of the best solution, 
while $\boldsymbol{\alpha}_{2}, \boldsymbol{\beta}_{2}$ is the probability amplitude of the current solution.

\section{QGA BASED MC-CDMA DETECTOR}

At the receiver, the transmitted data will be distorted by the effect of noise, fading, and multipath propagation, which necessitates the use of equalizing techniques to retrieve the original stream. As a first process in the MC-CDMA receiver, the cyclic prefix is discarded from the received packets. By performing this operation, any possible ISI or ICI will be eliminated. Then, the received data stream is converted to parallel form via S/P converter to be fed to the FFT block, where FFT process is done to the parallel streams. After the FFT process, the data is fed to the QGA processor where the equalization process is done to the received stream in order to retrieve the original stream. The QGA follows a certain procedure in order to find the solution and equalize the received stream. This procedure can be summarized by the following steps :-

Step(1) :- Initialize the operators $(\alpha, \beta)$ of all chromosomes of QGA at $t=0$ with the value of $1 / \sqrt{2}$ so the chromosomes will represent the linear superposition of all possible states. The Q-chromosome of $\mathrm{m}$ Q-genes will be expressed as shown in eq.(6) .

$$
\left|\psi_{q_{j}^{0}}>=\sum_{i=1}^{2^{n}} \frac{1}{\sqrt{2^{n}}}\right| R_{i}>
$$

Where $\left|\psi_{q_{j}^{0}}\right\rangle$ is the jth chromosome at $\mathrm{t}=0,\left|\mathrm{R}_{\mathrm{i}}\right\rangle$ is the ith state represented by a binary string $\left(\begin{array}{lllll}\mathrm{x}_{1} & \mathrm{x}_{2} & \mathrm{x}_{3} & \ldots & \mathrm{x}_{\mathrm{n}}\end{array}\right)$ where $\mathrm{x}_{\mathrm{k}}, \mathrm{k}=1,2,3, \ldots, \mathrm{n}$ is either 0 or 1 .

Step(2) :- Make a set of binary solutions $p(t)$ by observing the Q-chromosomes $\mathrm{q}(\mathrm{t})$ states where $\mathrm{p}(\mathrm{t})=\left(\mathrm{x}_{1} \mathrm{t}^{\mathrm{t}}\right.$, $\left.\mathrm{x}_{2}{ }^{\mathrm{t}}, \mathrm{x}_{3}{ }^{\mathrm{t}}, \ldots, \mathrm{x}_{\mathrm{m}}{ }^{\mathrm{t}}\right)$. each element of $\mathrm{p}(\mathrm{t})$ is a binary string of length $\mathrm{n}$ that can be extracted using the probability of the qubit $\left|\alpha_{i}^{t}\right|^{2}$ or $\left|\beta_{i}^{t}\right|^{2}, i=1,2,3, \ldots, n$.

Step(3) :- Evaluate the set of solutions based on the maximum likelihood rule and set the fittest chromosome as the target of next evolution values. The maximum likelihood rule is expressed in eq.(7) .

$$
E=\arg \min \|y-h . x\|^{2}
$$

Where $y$ is the received sample, $\mathrm{h}$ is the channel response matrix, $x$ is the possible solution provided by the QGA processor.

Step(4) :- store the fittest solution among $p(t)$ into $B(t)$ and perform the following loop :-

While (not-termination condition) do

Begin

$\mathrm{t}=\mathrm{t}+1$

Step(5) :- make a binary solution $p(t)$ by observing the $\mathrm{Q}(\mathrm{t}-1)$ states.

Step(6) :- evaluate $\mathrm{p}(\mathrm{t})$ via the stored maximum likelihood rule.

Step(7) :- update $Q(t)$ using quantum rotation gate.
Step(8) :- store the best solution among $B(t-1)$ and $p(t)$ into $\mathrm{B}(\mathrm{t})$.

$$
\text { Step(9) :- store the best solution b among B(t). }
$$

After finding the solution by QGA processor, the equalized stream is converted from parallel form to serial form using a parallel to serial converter. Then, the received stream is de-mapped by a certain de-mapping technique. By completing the de-mapping, the first stage of data reception is completed. Fig(3) shows the block diagram of the MCCDMA first stage.

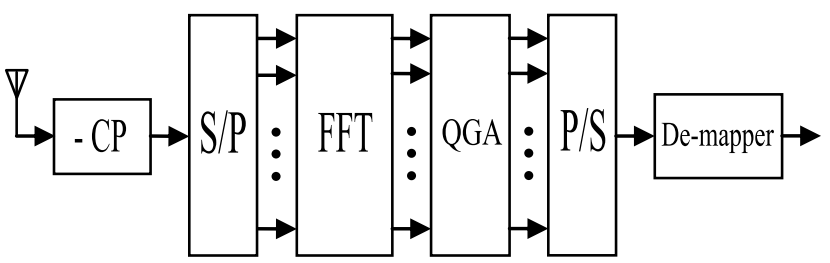

Figure 3: First Stage of MC-CDMA Receiver

After retrieving the original data stream, the main data stream is fed to the CDMA demodulator. Each user will extract his data stream from the main data stream by multiplying the main data stream by the same code used to spread his data stream at the transmission. After the multiplication, the resulted samples is summed and fed to a threshold detector in order to reconstruct the data stream for each user. Fig.(4) shows the structure of the second stage of data reception.

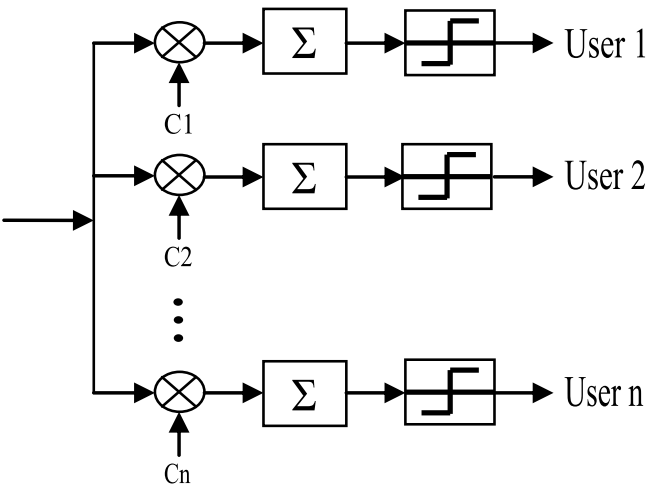

Figure 4: Second stage of MC-CDMA Receiver

Computer Simulations through MATLAB program were performed on the proposed system. The number of multiple users was 4 , the spreading code set was Walsh codes set, BPSK and QPSK were used respectively as mapping schemes, and a cyclic prefix of $25 \%$ was used to combat any possible ISI, ICI, and multipath effects. The bandwidth was divided to 16 sub-carriers through the use of 16 IFFT code. The channel characteristic was assumed to be unknown and a channel estimation process was performed through the use of pilot carriers.

For the QGA, the set of population was four chromosomes, the rotation angle was chosen to equal $0.005 \pi$ to ensure a steady convergence towards the solution.

In order to show the improvement in BER performance obtained by the use of QGA based MC-CDMA detector, a comparison was made with QGA based $4 \times 4$ MIMO-OFDM 
system proposed in previous work [4] for both BPSK and QPSK mapping schemes. Fig.(5) shows the performace results of the proposed QGA based MC-CDMA detector alongside with the QGA based MIMO-OFDM detector proposed in previous work for both BPSK and QPSK modulation schemes.

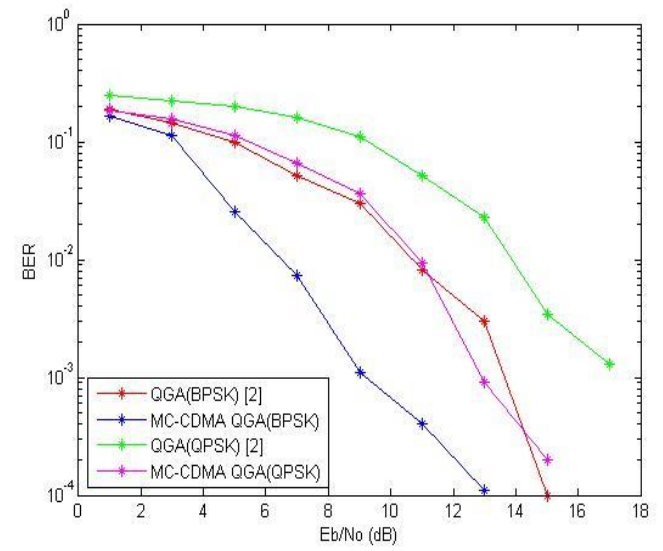

Figure 5: BER Performance for Various QGA Based Detector

\section{CONCLUSIONS}

In this paper, a detailed explanation on the MC-CDMA structure was given. the advantages of the MC-CDMA system over the conventional OFDM systems was stated.

The quantum genetic algorithm QGA was also explained in detail giving its procedure and the merits that make it outperforms the conventional GA.

The design of the QGA based MC-CDMA detector was discussed, the most important parameters for the proposed detector were given, and a comparison was held between the QGA based MC-CDMA detector and QGA based $4 \times 4$ MIMO-OFDM detector. The results show that the QGA based MC-CDMA detector has outperformed the QGA based
MIMO-OFDM detector proposed in previous work [4] by 5 $\mathrm{dB}$ for BPSK and $4 \mathrm{~dB}$ for QPSK, which proves that the MCCDMA system based on QGA for detection is better in BER performance than the QGA based MIMO-OFDM detector proposed in [4].

\section{REFERENCES}

[1] Mosa A. Abu-Rgheff. "Introduction to CDMA Wireless Communications", Academic Press, 2007.

[2] Mohammed Faisal, Jia Uddin, Iqbal H. Haider. "Simulation Based Performance Analysis of MC-CDMA and CDMA over Rayleigh Fading Channel", International Journal on Internet and Distributed Computing Systems. Vol: 2 No: 1, pp. 120-122, 2012

[3] Bhavana kulhare, and Poonam Sihna. "Simulation and Analysis of CDMA System under AWGN and Rayleigh Fading Channel", International Journal of Computers and Technology, vol.6, No.2, pp.336-342, 2013.

[4] F. Li, M. Zhou, and H. Li. "A novel neural network optimized by quantum genetic algorithm for signal detection in MIMO-OFDM systems", Proceeding of IEEE Global Telecommunications Conference,

[5] Z. Ge-xiang, L. Na, J. Wei-dong, and H. Lai-zhao. "Novel Quantum Genetic Algorithm and Its Applications", Frontiers of Electrical and Electronic Engineering in China, vol.1, Issue 1, pp.31- 36, 2006.

[6] F. Li, W. Wang, M. Zhou, and B. Y. Zheng. "Quantum Genetic Algorithm for Signal Detection in MIMOOFDM Systems", Proceeding of the 2010 International Conference on Genetic and Evolutionary Methods (GEM'10), USA, 2010

[7] F. Lei, and W. Wang. "Quantum Genetic Algorithm based Signal Detection Scheme for MIMO-OFDM System", Second International Conference on Communication Systems, Networks and Applications, Vol.1, pp. 298-301, Hong Kong, China, 2010 